\title{
MAIN CHAMBER INJECTORS FOR ADVANCED HYDROCARBON BOOSTER ENGINES*
}

\author{
Matthew R. Long ${ }^{\dagger}$, Vladimir G. Bazarov ${ }^{\ddagger}$, and William E. Anderson ${ }^{\S}$, \\ Purdue University, West Lafayette, IN, United States
}

\begin{abstract}
Achieving the highest possible specific impulse has long been a key driver for space launch systems. Recently, more importance has been placed on the need for increased reliability and streamlined launch operations. These general factors along with more specific mission requirements have provided a new focus that is centered on the oxidizer rich staged combustion (ORSC) cycle. Despite a history of use in Russia that extends back to the 1960's, a proven design methodology for ORSC cycle engines does not exist in the West. This lack of design expertise extends to the main chamber injector, a critical subcomponent that largely determines the engine performance and main chamber life. The goals of the effort described here are to establish an empirical knowledge base to provide a fundamental understanding of main chamber injectors and for verification of an injector design methodology for the ORSC cycle. The design of a "baseline" injector element, derived from information on Russian engines in the open literature, is presented. The baseline injector comprises a gaseous oxidizer core flow and an annular swirling fuel flow. Sets of equations describing the steady-state and the dynamic characteristics of the injector are presented; these equations, which form the basis of the design analysis methodology, will be verified in tests later this year. On-going cold flow studies, using nitrogen and water as simulants, are described which indicate highly atomized and symmetric sprays.
\end{abstract}

\section{NOMENCLATURE}

A - Swirl Injector Characteristic

AFRL - Air Force Research Laboratory

c* - Characteristic Velocity, $\mathrm{ft} / \mathrm{s}$

C - Emperical Constant

$\mathrm{C}_{\mathrm{D}}$ - Discharge Coefficient

$\mathrm{C}_{\mathrm{F}}$-Thrust Coefficient

d - Diameter, in

$\mathrm{d}_{32}$ - Sauter Mean Diameter, $\mu \mathrm{m}$

F-Thrust, lbf

\footnotetext{
* This work was funded by NASA Marshall Space Flight Center

†Graduate Student, School of Aeronautics and Astronautics,

Student Member AIAA

$\$$ Visiting Professor, School of Aeronautics and Astronautics

\$Assistant Professor, School of Aeronautics and Astronautics
}

$\mathrm{g}$ - Gravitational Acceleration, $\mathrm{ft} / \mathrm{s}^{2}$

$\mathrm{h}$ - Film Thickness, in

$\mathrm{I}_{\mathrm{sp}}$-Specific Impulse, sec

1 - Length, in

L'-Length from Injector to Throat, in

LOX - Liquid Oxygen

LRE-Liquid Rocket Engine

$M$ - Mass Flow Rate, lbm/s

MFR - Momentum Flux Ratio

$\mathrm{n}$ - Number of Tangential Inlets

NASA - National Aeronautics and Space Agency

NGLT - Next Generation Launch Technology

O/F - Oxidizer to Fuel Ratio

OMS-Orbital Maneuvering System

ORPB-Oxidizer Rich Preburner

ORSC - Oxidizer Rich Staged Combustion

P- Pressure, psi

PDPA - Phase Doppler Particle Analyzer

Q - Volumetric Flow Rate

RCS-Reaction Control System

$r$ - Radius of Tangential Orifice, in

$\mathrm{R}$ - Radius of Injector, in

RP-1 - Rocket Propellant Grade Kerosene

S- Surface Area, in ${ }^{2}$

St - Strouhal Number

T-Temperature, $\mathrm{F}$

$\mathrm{U}$ - Total Velocity at Exit, $\mathrm{ft} / \mathrm{s}$

$\mathrm{x}$ - Variable Length, in

Greek Letters

$\Pi$ - Injector Response Function

$\Sigma$ - Total

$\eta_{c^{*}}-C^{*}$ Efficiency

$\eta_{C F}-$ Thust Coefficient Efficiency

$\beta$-Degree coefficient

$\Delta$ - Change

$\rho$ - Density, $\mathrm{lbm} / \mathrm{in}^{3}$

$\varphi$-Coefficient of Nozzle Filling

$\sigma-$ Surface Tension, lbf/in

$\omega$ - Angular Frequency, /s

$\mu$-Dynamic Viscosity, lbf-s/in ${ }^{2}$

Subscripts

() ax - Axial

()$_{a v}$ - Average

() - Chamber

()e - Exit

( ) - Fuel

() - Gas

()$_{\text {in }}$ - Inlet to Nozzle

() $)_{l}$ - Liquid 
( ) $m$ - Closed Part of Vortex Chamber

()$_{m n}$ - Inside of Swirl Nozzle

()$_{n}$ - Nozzle

()$_{0}$ - Initial

()ox - Oxidizer

( $t_{t}$ - Throat

()$_{T}$ - Tangential Inlet

() $)_{\nu}$ - Vortex

() $)_{v n}$ - Free Vortex

() vac - Vacuum

\section{INTRODUCTION}

NASA is currently undertaking risk reduction activities focused on key technology areas to provide a basis for the successful development of oxidizerrich staged combustion (ORSC) cycle engines. One of these key technologies is the main combustion chamber (MCC). The MCC injector is a critical subcomponent that determines the performance, life, and combustion stability characteristics of the main combustor.

Injector design is a largely empirical endeavor, and is heavily reliant on design heritage. Injector types are usually designed specifically for an application, and are dependent on the propellant type and its state, and chamber geometry and operational requirements (flowrate, pressure drop, operating level range, life, etc.). A unique requirement for the ORSC cycle main chamber injector is that it uses the warm oxidizer exhaust from the preburner and a liquid hydrocarbon fuel. Very little experience with these types of injectors exists in the U.S.

Research is underway at Purdue University to develop a fundamental understanding of injector elements that are appropriate for use with the ORSC cycle engine. In addition to qualitatively characterizing the injector element, the approach taken to meet the primary objective is to obtain model validation data via the measurement of steady state performance parameters such as characteristic exhaust velocity, $\mathrm{c}^{*}$, and axial pressure gradient which can be related to the energy release profile; thermal parameters such as gas temperature and heat flux near the injector face and the chamber wall; and combustion dynamic characteristics.

The measurements will be taken at realistic single element geometric scales and operating conditions. Conditions for the design analysis are derived from open-literature information on the ORSC cycle. Parametric analyses and cold flow studies are underway to evaluate the effects of element geometry and operation on steady state combustion, heat transfer, and combustion dynamics.
This paper provides a current status on this activity at Purdue. First, a background of injector types that have been used in the ORSC cycle is provided. A rationale is presented for determining the initial element geometry and operating conditions that will be tested. The equations used to design the injector will be described, and the "baseline" design is presented. Results to date from cold-flow studies are described. Finally, conclusions from the analyses and experiments conducted to date are summarized.

\section{HISTORY OF THE ORSC CYCLE AND ITS MAIN CHAMBER INJECTORS}

Design of ORSC cycle engines using LOX/kerosene propellants began in Russia in 1959. Since then more than 26 liquid rocket engines (LRE's) with thrust levels from $4500 \mathrm{lbf}$ up to $1,868,200 \mathrm{lbf}$ have been developed. ${ }^{1} \quad$ The most well-known ORSC cycle engines are the NK family, developed in $1968-72$ by Samara Design \& Production enterprise: the singlechamber NK-33 (Fvac= 368,000 lbf, Isp= $331 \mathrm{sec}$, $\mathrm{Pc}=2150$ psia); the NK-43 (Fvac $=394,517 \mathrm{lbf}$, Isp= $346 \mathrm{sec}, \mathrm{Pc}=2113$ psia); and the NK-39 (Fvac=91,497 lbf, Isp=352 sec, $P_{c}=1360$ psia). These engines were used for the $1^{\text {st }}, 2^{\text {nd }}$, and $3^{\text {rd }}$. stages, respectively of the N-1 (Science-1) launcher. Although this program was cancelled in 1972, the engines had already been fully developed.

Most of the ORSC cycle engines used presently were designed and produced by the Energomash enterprise in Khimki, Russia. These engines are the singlechamber RD-120, developed in 1976-85 for the 2nd stage of the "Zenit" rocket (Fvac $=187,266 \mathrm{lbf}$, Isp=350 sec, $\mathrm{Pc}=2350 \mathrm{psia}$ ); the four-chamber RD170 (Fvac $=1,776,670 \mathrm{lbf}$, Isp= $337 \mathrm{sec}, \mathrm{Pc}=3553$ psia) for the 1st stage of the Energia launcher and the Zenit-3SL rocket; the two-chamber RD-180, mutually developed in 1994-98 by Energomash and Pratt \& Whitney for the Atlas-3A and Atlas-5 missiles (Fvac $=933,407 \mathrm{lbf}$, Isp $=337.8 \mathrm{sec}, \mathrm{Pc}=$ 3,771 psia.); and the single-chamber RD-191 (Fvac= $467,603 \mathrm{lbf}, \mathrm{Isp}=337 \mathrm{sec}, \mathrm{Pc}=3,800$ psia) just now under development for the new Russian "Angara" commercial launcher.

Many valuable results with respect to the ORSC cycle were gained by the Energia enterprise in Korolev, which developed the medium-thrust RD-58 engine (Fvac $=19,109 \mathrm{lbf}$, Isp= $361 \mathrm{sec}, \mathrm{Pc}=1131$ psia), which served as an experimental model to scrutinize the ORSC cycle. This engine and its modifications have high records of reliability and are widely used, as in the final stage of the $\mathrm{N}-1$, the Proton, the Energia, the Zenit-3, the Block-D of 
Zenit-3MSL, as well as a part of the integrated OMS/RCS system of the Buran space plane. ${ }^{2}$

The wide use of this cycle was motivated by its obvious advantages: non-toxic and inexpensive propellants (liquid oxygen and kerosene), high density specific impulse, and high cycle efficiency. Of course, the high value of combustion chamber pressure was not simple to achieve. Many problems appeared - combustion chamber wall and injector face cooling, high pressure turbomachinery and, naturally, problems with combustion stability, which have been hypothesized to grow with the combustion chamber pressure to the third degree. ${ }^{3}$ Considerable effort was spent to produce engines with the required durability, efficiency and life.

A primary objective of this study is to experimentally characterize the steady state and dynamic characteristics of a representative ORSC cycle MCC injector. Due to the plurality of staged combustion cycle LRE built in Russia, practically all possible types of gas-liquid injectors were tested, developed and used in these engines. A review of these injector types is presented here.

These injectors can be classified by the relative orientation of the oxidizer and fuel flows (inside/outside) and whether the flows are swirled.

Gas from outside, liquid from inside In the so-called shear coaxial injector, neither propellant flows are swirled. Advantages of shear coaxial injectors are in their simplicity, ability to be placed closely near by each other, and rather good atomization and uniformity of combustible mixture. They have been widely used in staged combustion cycle engines, mainly using $\mathrm{LOX} / \mathrm{H} 2$ such as Vulcain for the Ariane 5, the RD-0120 for the Energia launcher and the Space Shuttle Main Engine. Disadvantages of the shear coaxial injector, as latest studies showed, ${ }^{4}$ are in their sensitivity to pressure drop pulsation and, moreover, their ability to generate self-oscillations both of the liquid and two-phase flows. These selfoscillations, even if they do not cause combustion instability, lead to unevenness of combustible mixture and so-called "vibro-activity" of combustion. The elevated noise amplitude can cause fatigue cracks in high pressure combustion chambers during long life work.

In a "swirl coaxial" injector element, the swirling interior liquid propellant is placed coaxially in an exterior gas channel. The swirling liquid flow forms a conical hollow film, which is injected into the surrounding gas flow and is atomized. This feature eliminates the unsteadiness of two-phase flow in shear coaxial injectors. Advantages of these injectors are its much better and uniform atomization that allows a high mass flow rate injector element.

Disadvantages of this injector type are in its more complicated design, manufacturing, and in its ability, if not properly designed, to form strong, regular selfoscillations of the liquid film in coaxial gas flow. ${ }^{5}$ These injectors are typically used in LOX/H2 engines such as the RD-57 and the RL- 10 .

Bicentrifugal coaxial swirl injectors swirl both propellants. These injectors provide very high atomization and mixing efficiencies and therefore were whatefy used in medium and low thrust gasliquid combustion chambers using $\mathrm{LOX} / \mathrm{H} 2$ or LOX/kerosene propellants, such as the Buran spacecraft OMS/RCS engines. ${ }^{2}$

The main disadvantage of such injectors is in its high sensitivity to disturbances of the combustion chamber pressure and velocity and relatively poor thermal protection of the injector face, which hindered their use in high pressure, large thrust combustion chambers.

Gas from inside, liquid from outside Injector elements with an inner gas core surrounded by liquid fuel include two types - the first where liquid is injected into the central gas stream through radially drilled orifices and the other where the liquid is injected through tangential orifices to provide an annular centrifugal flow. A typical injector of the first type comprises a hollow tube for gas flow with a number of drillings in the tube walls. When liquid is supplied under excess pressure, liquid sprays are formed and bent in the gas flow. The distance between the drillings and gas tube exit can be different, as can be the inclination angle of axis of the liquid orifice to the injector axis. The latest fashion of their design comprise so-called "hordal" channels, in which the axes of the penetrating jets do not cross the axis of the gas pipe. Such injectors provide the most uniform mixing.

Advantages of such injectors are in their simplicity, good atomization and mixing, the possibility to tune the acoustic characteristics of gas stage, and design heritage and maturity. Such injectors were widely used in medium thrust middle-pressure combustion chambers using hypergolic propellants. Disadvantages are the high sensitivity of the injector to pressure and velocity disturbances in the combustion chamber due to free atomization of liquid sprays by the gas flow. The combustion zone is 
attached closely to the injector exit and reverse flows around the injector bring hot gases to the injector face. Therefore, when using such type of injectors, special means to protect the injector face from overheating must be used such as additional low mass flow rate fuel swirl injector around the injector nozzle.

The second type of injector - the axial gas core/centrifugal liquid annulus - usually comprises a tube for the axial gas flow that includes an openended vortex chamber of a liquid swirl injector which serves simultaneously as a mixer of gas and liquid flows. Liquid propellant is supplied by a number of tangential channels, manufactured usually in two rows. If the size of the injector allows, the initial part of the liquid flow is separated from the gas flow by a collar to provide angular uniformity of atomized flow. The mechanism of atomization is still of Kelvin - Helmholtz type, but liquid is stabilized by centrifugal forces along the wall and can be atomized only by the formation of surface waves. Due to a much larger liquid surface area compared to shear coaxial injectors, and thinner liquid flow thickness, atomization is improved which allows injector elements with greater mass flow rates per element.

The advantages of such an injector type are in its significantly lower sensitivity to combustion chamber pulsations due to the stabilization of the liquid film on the surface of the vortex chamber wall. The injector usually forms fuel-enriched flow and even liquid fuel film at the periphery of the vortex chamber edge and thus provides necessary cooling and protection of the injector face from the hot products of combustion. The gas cavity usually also acts as an acoustic resonator to withdraw a significant part of acoustic energy from the combustion chamber to the gas manifold. In order to improve atomization, the gas also can be swirled, and the gas vortex chamber with its tangential inlets will thus serve as Helmholtz resonator.

The disadvantages of this kind of injector are its more complicated design as evidenced by the calculation of steady-state and dynamic characteristics, and poor atomization at low pressures and gas densities. But for high chamber pressures typically encountered in the ORSC cycle, this injector works well and thus became the main type of injector for high thrust, high pressure combustion chambers for ORSC cycle LOX/kerosene LRE's such as the RD-120, RD-170, and RD-180.

The great potential of gas-centered liquid swirl injectors has inspired new research and development efforts within the West. Recently, Sierra Engineering partnered with the Air Force Research Laboratory Propulsion Directorate to study gas-centered liquid swirl injectors. The study focused on development of a small combustion chamber to evaluate alternative hydrocarbon fuels for the Air Force Research Laboratory. Three injector variations were conceived and tested: converger, diverger, and prefilmer, classified according to the injector element exit geometry. Cold flow testing revealed that each of the variations produced fine atomization and a uniform spray. The injectors also produced mean drop sizes on average 1/3 - 1/4 of equivalent shearcoaxial injectors. While $\mathrm{c}^{*}$ efficiencies above $90 \%$ were observed, some injectors exhibited a "chug" instability at different operating conditions. 7.8

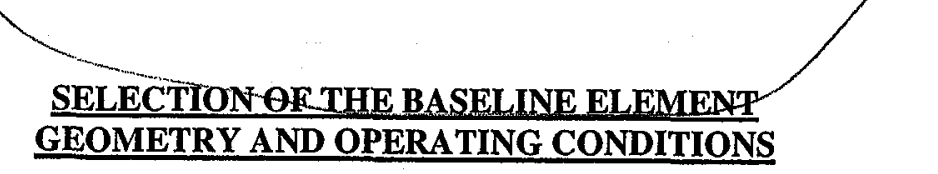

The first step in the injector characterization is the selection of a "baseline" element. The requirements for the baseline element are that it is representative of element types that are used in ORSC main chambers, and that its specific design and operating parameters can be derived from the open literature. The injector element is also designed to be modular so that variations in design parameters can be evaluated relatively easily.

The operating parameters for a well-known ORSC cycle engine - the RD-170 - were summarized by Manski, et al. ${ }^{9}$ Main chamber design parameters were summarized by Sutton and Biblarz. ${ }^{10}$ Sketches of an injector for a ORSC cycle MCC, with important design features, were provided in a U.S. Patent application submitted by Katorgin, et al. ${ }^{11}$ These three sources provided the basis for the design of the baseline element.

First the element size was scaled from the patent information, which indicated a total of 271 elements, including baffle and near-wall elements. Using the single-chamber thrust level of the RD-170 $(\sim 465,000$ lbf), the thrust-per-element was initially determined to be about $1700 \mathrm{lbf}$. The chamber pressure of the RD-170 main chamber is 3720 psia, however the test operating pressure of 2150 psia was chosen to be a safe operating level with the propellant supply pressures available at the Maurice Zucrow High Pressure Laboratory at Purdue, where testing will occur. 
The single element test combustion chamber is designed to approximate the conditions a single element would see in a full scale engine. Chamber sizing was determined by considering contraction ratio and cross-sectional area-per-element. The combination of single element thrust level and chamber pressure resulted in a throat diameter of about $0.7 \mathrm{in}$; however, this throat diameter was deemed to be too small for reliable manufacturing and practical cooling methods, so the throat diameter was increased and set arbitrarily at $1.0 \mathrm{in}$, which resulted in a single element vacuum thrust level of $3000 \mathrm{lbf}$. The resultant chamber contraction ratio was 2.25 , which is considered sufficiently close to the RD-170 contraction ratio of 1.61 . The nozzle expansion ratio was chosen to expand the combustion gases to atmospheric pressure. The chamber and nozzle parameters are summarized in Table 1.

Table 1: Chamber and Nozzle Dimensions

\begin{tabular}{|c|c|}
\hline \multicolumn{2}{|c|}{ Chamber Dimensions } \\
\hline $\mathrm{P}_{\mathrm{c}}$ (psia) -Throat & 2150 \\
\hline$\varepsilon_{\mathrm{c}}$ & 2.25 \\
\hline $\mathrm{d}_{\mathfrak{t}}$ (in) & 1.0 \\
\hline $\mathrm{d}_{\mathrm{c}}$ (in) & 1.5 \\
\hline $\mathrm{L}^{\prime}$ (in) & 12 \\
\hline \multicolumn{2}{|c|}{ Nozzle Dimensions } \\
\hline $\mathrm{d}_{\mathrm{t}}$ (in) & 1.0 \\
\hline$\varepsilon_{\mathrm{n}}$ & 17.2 \\
\hline $\mathrm{d}_{\mathrm{e}}$ (in) & 4.15 \\
\hline
\end{tabular}

The element $\mathrm{O} / \mathrm{F}$ and oxidizer properties were determined from the RD-170 cycle data provided by Manski et al. ${ }^{9}$ The composition and properties of the combustion gas from the oxidizer-rich preburner (ORPB) were determined first. The ORPB exhaust temperature is approximately $750^{\circ} \mathrm{F},{ }^{9}$ which determines the $\mathrm{O} / \mathrm{F}$ of the preburner and the composition of the oxidizer-rich gases entering the injector. The NASA-Lewis Isp code was used to determine the optimum injector $\mathrm{O} / \mathrm{F}$, determining the $c^{*}$ and $\mathrm{C}_{\mathrm{Fvac}}$. The frozen and equilibrium flow parameters were averaged to calculate the Isp $p_{\text {vac }}$ as shown in Eq. 1. The propellant mass flow rates were then chosen to produce a thrust of approximately $3000 \mathrm{lbf}_{\mathrm{v}}$. A modest chamber pressure of 2150 psia was chosen for facility compatibility and safety reasons. The operating conditions are summarized in Tables 2 and 3.

$$
I s p_{v a c}=\frac{\eta_{c^{*}} c^{*} \eta_{C_{F v}} C_{F v}}{g}
$$

Table 2: Oxidizer-Rich Preburner Operating Conditions

\begin{tabular}{|l|c|}
\hline $\mathrm{O} / \mathrm{F}$ & $55.5: 1$ \\
\hline $\mathrm{M}_{\mathrm{ox}}$ (Lox) (lb/s) & 6.89 \\
\hline $\mathrm{M}_{\mathrm{f}}(\mathrm{RP}-1)(\mathrm{lb} / \mathrm{s})$ & 0.11 \\
\hline $\mathrm{T}_{\mathrm{c}}$ (F) & 746 \\
\hline Combustion Products By Weight & \\
\hline $\mathrm{CO}_{2}$ & $5.6 \%$ \\
\hline $\mathrm{H}_{2} \mathrm{O}$ & $2.2 \%$ \\
\hline $\mathrm{O}_{2}$ & $92.2 \%$ \\
\hline
\end{tabular}

Table 3: Main Injector Operating Conditions

\begin{tabular}{|l|c|}
\hline$P_{c}$ (psia) - Injector Face & 2263 \\
\hline$P_{c}$ (psia) - Entrance to Throat & 2150 \\
\hline $\mathrm{C}_{\mathrm{Fv}}$ & 1.83 \\
\hline$\eta_{\mathrm{CFv}}$ & 0.98 \\
\hline $\mathrm{c}^{*}(\mathrm{ft} / \mathrm{s})$ & 5860 \\
\hline$\eta_{\mathrm{c}^{*}}$ & 0.97 \\
\hline Isp $\mathrm{vac}(\mathrm{sec})$ & 317.0 \\
\hline$\Delta \mathrm{P}_{\mathrm{ox}}(\mathrm{psid})$ & 226 \\
\hline$\Delta \mathrm{P}_{\mathrm{ff}}(\mathrm{psid})$ & 226 \\
\hline $\mathrm{U}_{\mathrm{ox}}(\mathrm{ft} / \mathrm{s})$ & 400 \\
\hline $\mathrm{U}_{\mathrm{f}}(\mathrm{ft} / \mathrm{s})$ & 204 \\
\hline $\mathrm{F}_{\mathrm{vac}}\left(\mathrm{lbf} \mathrm{v}_{\mathrm{v}}\right)$ & 2991 \\
\hline $\mathrm{O} / \mathrm{F}$ & $2.87: 1$ \\
\hline $\mathrm{M}_{\mathrm{ox}}(\mathrm{Ox}-\mathrm{Rich} \mathrm{Gas})(\mathrm{lb} / \mathrm{s})$ & 7.00 \\
\hline $\mathrm{M}_{\mathrm{f}}(\mathrm{RP}-1)(\mathrm{lb} / \mathrm{s})$ & 2.44 \\
\hline $\mathrm{T}_{\mathrm{c}}(\mathrm{F})$ & 6431 \\
\hline
\end{tabular}

Next the baseline operating conditions were used to size the injector using a 1-D design code written in MathCad. The central bore passage for the oxidizerrich gas was determined by the design pressure drop 
taking into account real gas and dynamic compressibility. The oxidizer-rich gas pressure drop is determined by two notches placed at the inlet to the injector, as observed in the RD-170 patent. ${ }^{11}$ It is speculated that the purpose of the notches is to set the effective acoustic length of the injector.

The next step was to design the fuel swirl cup part of the injector. Another feature observed in the patent is the presence of a swirl collar concentric with the fuel tangential inlet ports. The presence of the collar serves to protect the fuel film from the high velocity oxidizer-rich gas, permitting it to form an intact annular film before it is exposed to the high velocity oxidizer flow.

\section{INJECTOR DESIGN ANALYSIS}

A survey of classical swirl injector theories and a comparison of their predictions to cold flow testing was recently undertaken. ${ }^{12}$ Based on this study, the swirl injector model presented by Bayvel ${ }^{13}$ was adapted to design the fuel swirler section of the injector. Since the vortex chamber diameter and outlet diameter are set equal (straight bore), the fuel swirl injector design is simplified, yielding only one solution from swirl injector theory for a given combination of propellant mass flow and pressure drop.

Next, the atomization processes were examined. According to the general atomization theory for the Kelvin-Helmholtz atomization mechanism, the mean droplet diameter (neglecting liquid viscosity) can be defined as

$$
\left(d_{32}\right)_{\mu=0}=C_{1} \frac{\sigma_{l}}{\left.\rho_{g} \mid \vec{U}_{g}-\vec{U}_{l}\right)^{2}}
$$

where $C_{l}$ is an empirical constant, and $\left|\vec{U}_{g}-\vec{U}_{l}\right|$ is the module of the vector difference between gas and liquid velocities. For shear coaxial injectors, $C_{l}=62$.

Taking into account the liquid viscosity $\mu_{l}$,

$$
d_{32}=\left(d_{32}\right)_{\mu=0}\left[1+C_{2}\left(\frac{\mu_{l}^{2} \rho_{g}}{\sigma_{l}^{2} \rho_{l}}\right)^{1 / 4} \cdot\left(\left|\vec{U}_{g}-\vec{U}_{l}\right|\right)^{1 / 2}\right]
$$

Where $C_{2}$ is an empirical constant, and for shear coaxial injectors is equal to 0.035 .
The mass flow rate of the atomized liquid per unit of liquid surface can be defined in the inviscid case as:

$$
\left(\frac{\partial M}{\partial S}\right)_{\mu=0}=C_{3}\left|\vec{U}_{g}-\vec{U}_{l}\right| \sqrt{\rho_{g} \cdot \rho_{l}}
$$

Where $C_{3}$ is an empirical constant, and for shear coaxial injectors is equal to 0.17. Taking into account liquid viscosity will give:

$$
\begin{aligned}
& \frac{\partial M}{\partial S}=\left(\frac{\partial M}{\partial S}\right)_{\mu=0} . \\
& {\left[1+C_{4}\left(\frac{\rho_{g}}{\rho_{l}}\right)^{1 / 4} \cdot\left(\frac{\left|\vec{U}_{g}-\vec{U}_{l}\right| \cdot \mu_{l}}{\sigma_{l}}\right)^{1 / 2}\right]}
\end{aligned}
$$

where $C_{4}$ is an empirical constant equal to -0.16 for shear coaxial injectors.

The aim of experimental research with basic injector is to verify if these constants are valid for atomization of liquid circular film and to define corrected values of $C_{1}$ through $C_{4}$.

Let us define the change in liquid film thickness due to atomization of liquid by gas flow. Approximately considering gas and liquid velocities to be constant along the mixing zone,

$$
h_{l}=h_{o}-x \frac{\partial M}{\partial x} / U_{a l} \rho_{l}
$$

where $h_{o}$ is the initial liquid film thickness, $x$ is the variable length, and $U_{a l}$ is the axial velocity of liquid film. The length of the mixer filled by liquid film can be defined as the value of $x$ where $h_{l}=0$ :

$$
\text { . } \frac{x}{h_{o}}=\frac{U_{a l} \cdot \rho_{l}}{\partial M / \partial s}
$$

\section{Injector Dynamics}

The specific combusting flow conditions in main combustion chambers, such as high pressure and gas density, high heat release per unit of volume, low combustion product residence time, low injector pressure drop (relative to combustion chamber pressure) and subsequent high response on pressure fluctuations provide immense role of injection as a governing process which defines combustion efficiency and stability. The stationary characteristics of an injector in an LRE define the uniformity : of propellant mixing, and the completeness and dynamic characteristics of combustion, especially the sensitivity of the flame to 
pressure and velocity pulsations. It has also been shown ${ }^{14}$ that the injector fulfills functions of a sensitive element, amplifier, phase shifter and actuator, as it is able to respond to pressure drop pulsations through the modulation of certain output parameters (mass flow rate, angle of spray, mean droplet diameter and spectrum of droplets, O/F ratio). The response is shifted in time to some angle that depends on inertia of the flow inside the injector. In some cases, the injector, as a means of two-phase flow formation, is able to generate self-oscillations. ${ }^{14}$

The baseline injector is shown in Fig. 1. The gas stage of the injector comprises a cylindrical tube with an orifice at the entrance and a two-phase flow generator at the outlet. Acoustic characteristics of such pipes were well studied. ${ }^{15}$ It is characterized by the impedance, or acoustic resistance, that comprises a complex relation between the sound pressure $p^{\prime}$ and the velocity pulsations $w^{\prime}$. As an acoustic resonator, the gas side of the injector comprises an open tube on each end, acting as a half-wave resonator. When properly tuned, it withdraws a significant part of the acoustic energy from the combustion chamber to the gas manifold, which can be dissipated by perforated plates.

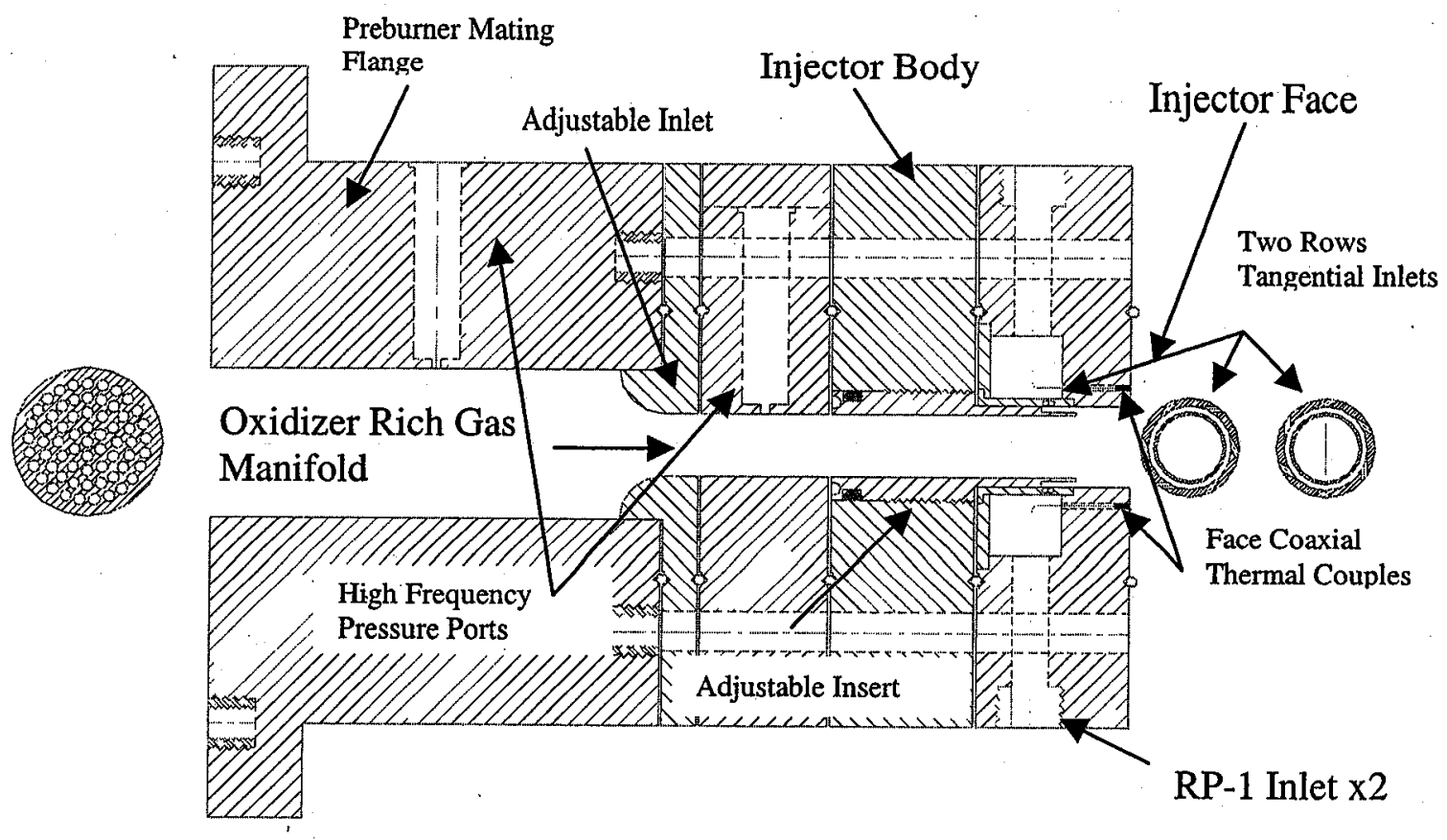

Figure 1: Baseline Injector Design

The liquid site of the injector comprises the so called "open" swirl injector with two rows of tangential channels in the bottom section and a vortex chamber, the opened orifice of which serves as the swirl injector nozzle. Steady parameters of such an injector can be defined by classic swirl injector theory. ${ }^{13}$ Peculiarities of stationary operation of the open swirl injector are more sensitive to inaccuracy if its production that will cause non-uniformity of liquid flow inside and at the exit of the vortex chamber.

The radius of the liquid vortex in the closed part of the vortex chamber, where $U_{a}=0$, is defined as

$$
r_{m k}=\frac{(1-\varphi) \sqrt{2}}{\sqrt{2-\varphi}} \cdot R_{n}=R_{n} \sqrt{a}
$$

where $\varphi$ is the relation of the nozzle area $\left(\frac{\pi\left(R_{n}^{2}-r_{m n}^{2}\right)}{\pi R_{n}^{2}}\right)$, filled by the liquid, to the total nozzle area. It is defined from the injector geometrical characteristic $A$ :

$$
A=\frac{(1-\varphi) \sqrt{2}}{\varphi \sqrt{\varphi}}
$$


where $A=\frac{R_{i n} R_{n}}{n r_{i n}{ }^{2}}$ is the non-dimensional criteria of similarity of a swirl injector outflow characteristics. The radius of the liquid film in the nozzle $r_{m n}$ :

$$
r_{m n}=\sqrt{1-\varphi} \cdot R_{n}
$$

and the axial velocity in the nozzle can be defined as:

$$
U_{a n}=\sqrt{\frac{\varphi}{2-\varphi}} \cdot U_{\Sigma}
$$

where $U_{\Sigma}=\sqrt{2 \Delta p_{\Sigma} / \rho}$

The axial velocity in the vortex chamber $W_{a y}$ can be defined as:

$$
U_{a \nu}=\frac{\mu}{\sqrt{1-a}} U_{\Sigma}=\frac{\varphi}{\sqrt{3-2 \varphi}} \cdot U_{\Sigma}
$$

and the corresponding value of the liquid vortex radius $r_{m}$ :

$$
r_{m}=\sqrt{\frac{a}{1-\bar{U}_{a \nu}^{2}}} \cdot R_{n}=\sqrt{\frac{a}{1-\frac{\varphi}{3-2 \varphi}}} \cdot R_{n}
$$

At the edge of the opened vortex chamber, the centrifugal pressure of the rotating liquid film will be transformed to axial acceleration of the liquid flow and axial velocity will be increased:

$$
U_{a_{e x}}=U_{a n} \sqrt{3-2 \varphi}
$$

The radius of liquid film will also be increased according to the mass conservation equation:

$$
r_{m_{e x}}=\sqrt{1-\frac{\varphi}{\sqrt{3-2 \varphi}}} \cdot R_{n}
$$

So, even without the influence of the gas flow, the liquid film thickness in the vortex chamber of open swirl injector will be decreasing from the place of inlet orifice towards the exit of the vortex chamber.

Having defined the steady state characteristics, the next task is to properly address the dynamic characteristics of the injector. The response function of a liquid injector can be defined according to a combination of its parts: ${ }^{16}$

$$
\Pi_{\Sigma}=\frac{Q_{n}^{\prime} / Q_{n}}{\Delta \rho_{\Sigma}^{\prime} / \Delta \rho_{\Sigma}}=\frac{\bar{R}_{\nu}^{2}}{a} \cdot \frac{\Pi_{T} \cdot \Pi_{v n} \cdot \Pi_{n}}{2 \Pi_{T} \cdot \Pi_{v c}-1}
$$

where $\Pi_{T}$ is a complex response function of tangential channels:

$$
\Pi_{T}=\frac{Q_{T}^{\prime}}{Q_{T}} / \frac{\Delta p_{T}^{\prime}}{\Delta p_{T}}
$$

$\Pi_{v n}$ is the complex response function of the vortex chamber:

$$
\Pi_{v n}=\frac{Q_{v n}^{\prime}}{Q_{v n}} / \frac{Q_{T}^{\prime}}{Q_{T}}
$$

$\Pi_{n}$ is a complex response function of the nozzle that in the case of opened injector comprises a part of the vortex chamber:

$$
\Pi_{n}=\frac{Q_{n}^{\prime}}{Q_{n}} / \frac{Q_{v n}^{\prime}}{Q_{v n}}
$$

$\Pi_{v c}$ is the response function of the closed end of the vortex chamber and comprise a feed back connection:

$$
\Pi_{v c}=\frac{\Delta p_{v c}^{\prime}}{\Delta p_{T}} / \frac{Q_{T}^{\prime}}{Q_{T}}
$$

The peculiarities of the response function of the baseline swirl injector can be easily seen from the Eq. (16). The coefficient $\bar{R}_{v}^{2} / a$ has a minimum value when $\bar{R}_{v}^{2}=1$.

So, the open swirl injector has the lowest response from all other types of swirl injectors. Due to the absence of any reflected waves from the opened end, no resonance conditions will be in the vortex chamber and the nozzle. Therefore, for $\Pi_{v n}$ and $\Pi_{n}$ definition, conditions are strongly simplified. In the conditions of the ideal liquid, values of their modules will be equal to 1 and these elements will serve only as phase shifters. As to $\Pi_{v c}$ - differently from classical, "closed" swirl injectors - the thickness of liquid film is comparable with the diameter of tangential channels and all the film in the closed end will have pulsation of circumferential velocity, synchronous with the pulsation of the flow in tangential channels ( $1^{\text {st }}$ model in general dynamics theory ${ }^{16}$ ), and the response of the vortex chamber flow can be considered as quasi-stationary. This fact strongly simplifies all the calculations of the opened swirl injector response. Visa versa, the existence in the vortex liquid motion of two mechanisms of disturbances propagation mentioned in $^{14}$ (one by means of surface waves propagation, and the other by means of axial spreading of liquid with periodically changed vorticity) will make the definition of the 
nozzle dynamic characteristics more complicated as velocities of their movement are quite different, depending on centrifugal acceleration and liquid film thickness which will change along the vortex chamber under the influence of the gas flow. For real conditions they are not yet studied.

For ideal liquid and absence of gas flow, the wave velocity is twice the axial velocity of liquid film and therefore it could be expected that some length of the vortex chamber will exist where both mechanisms will give an equal signal in opposing phases and will suppress each other. So, the main peculiarity of the open swirl injector in the dynamic sense is its possibility to filter pulsations of certain frequencies from all the spectrum of pulsations and to suppress the output pulsations of the mass flow rate. For the main combustion chamber injector it may be insignificant as practically all liquid from liquid vortex will be atomized by gas flow, but for liquidliquid gas generator injectors it will be very significant and needs to be studied. The expected length of the vortex chamber that will suppress flow pulsation can be defined from its Strouhal number $S t$ $=L_{n} / \lambda_{w}$, for the basic injector $L_{n}$ is about $14.5 \mathrm{~mm}$.

As to $\Pi_{T}$, it also has some peculiarities. As there are two rows of tangential channels with about four $\mathrm{mm}$ between them, initial disturbances of velocity waves for low amplitudes can be calculated independently according to the methodology presented in ${ }^{16}$ for arbitrarily placed tangential channels. The axial velocity between them is twice less than in the rest of the vortex chamber. Half the wave length will approximately be equal to $4.35 \mathrm{~mm}$ for the frequency of $1200 \mathrm{~Hz}$, and circumferential velocity fluctuations from each row will be in opposing phases and will completely suppress each other.

The effect of liquid flow pulsation on the dynamics of atomization and mixing is dominated by the simultaneous atomization of liquid portions injected at different time and having different mass flow rates, corresponding to the moment of their injection in the vortex chamber. The result of such interaction will be a strong decrease of the response function module via growth of the Strouhal number of the mixing zone $S t_{m}=\omega L_{n} / U_{a l}$. This influence is similar to the mechanism of flow pulsation integration during combustion, described by Bazarov, ${ }^{17}$ and can provide total insensitivity of the injector to any pulsation of liquid flow.

The effect of a gas flow pulsation on the dynamics of atomization and mixing is that it will excite a pulsation of the atomized liquid. The total sensitivity of the injector to gas pulsation is defined as: ${ }^{4}$

$$
\left|\Pi_{a g}(\omega)\right|=\frac{\beta \omega L_{v c}}{3 U_{g}}
$$

where $\beta$ is a degree coefficient in the expression of atomization velocity; usually it is less then one and depends on the mechanism of atomization. As the gas velocity is much higher than the axial velocity of the liquid, the response of the atomized mass flow rate will be rather low. Not so with the sensitivity of gas flow in injector; this will be significant. Therefore, under the influence of pressure disturbances in the combustion chamber, the injector will generate pulsations of $\mathrm{O} / \mathrm{F}$ ratio. Such nonstoichiometric composition will burn for more long time and occupy more length of the combustion chamber. The sensitivity of the combustion zone to any fluctuations of mass flow rate and combustion chamber pressure will be decreased. So, the studied injector is expected to comprise not only linear damper and suppressor of liquid flow pulsation, but also as non-linear servo-stabilizer of the operational process. The process of atomization of liquid film, stabilized by vortex chamber walls, is a relatively stable process with respect to the development of self-oscillations. No self-oscillations were experimentally found out in such injectors, differently from shear/coax and others with the internal liquid flow.

\section{COLD FLOW TESTS}

Cold flow testing was conducted at the Air Force Research Lab (AFRL) High Pressure Cold-Flow Injector Characterization Laboratory. The injector consisted of an acrylic body (seen in Fig. 2) and acrylic injector inserts. Acrylic was chosen to enable the observation of the film formation and atomization within the injector. The threaded inserts fastened into the bottom of the acrylic body and permitted simple alteration of the swirl injector and gas bore geometry. Three diagnostic techniques were used on the resulting spray: flow visualization by back-lit photography, spray patternation, and laser diagnostics techniques by use of a Phase Doppler Particle Analyzer (PDPA).

A parametric test matrix was devised to scale the physics that dominate atomization within the full scale injector. After evaluating a number of traditional non-dimensional parameters, two parameters were best suited to describe the spray: the differential velocity of the gas and liquid film and total momentum flux ratio. 
The differential velocity was defined as the difference between the gas axial velocity and the liquid film velocity:

$$
\Delta U=\left|U_{o x_{a x}}-U_{f_{a x}}\right|
$$

with water and nitrogen were used as the fuel and oxidizer simulants respectively.

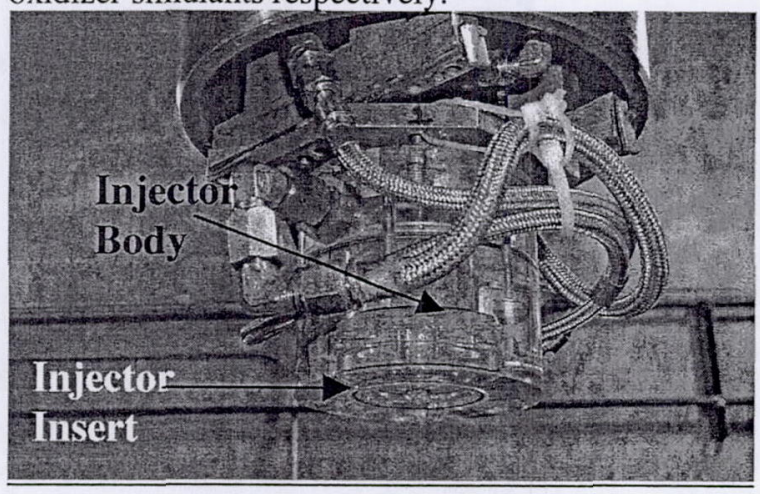

Figure 2: Acrylic Injector Body Installed at AFRL

The momentum flux ratio (MFR) is defined as:

$$
M F R=\frac{\rho_{f} U_{f}^{2}}{\rho_{o x} U_{o x}^{2}}
$$

The differential velocity survey was conducted to evaluate the effects of the gas shearing force on the spray formation at two chamber pressures (300 and 1150 psia) with an injector insert for each condition. The velocities of the simulants are then varied by adjusting the mass flow rates through a fixed injector insert. The chamber pressure of 1150 psia was designed to have the nitrogen density approximately that of the oxidizer-rich gas in the full scale element. However, since the dense sprays at pressure could make it difficult to penetrate with a PDPA, a second test series was designed at a lower chamber pressure of 300 psia to permit the full range of diagnostic tools to be used on a less dense spray.

Concurrent with the differential velocity survey, qualitative observations were made on the liquid film, and the processes by which it is entrained into the gas stream. From the drawings illustrated in the Figs. 4 and 7, the swirled liquid is injected directly into the gas bore without a collar as in the baseline. With only the liquid flow, the film backflows into the gas plenum, until an equilibrium point is reached, and flows out normally producing a conical sheet as predicted by classical swirl injector; this can be see in Figure 3.

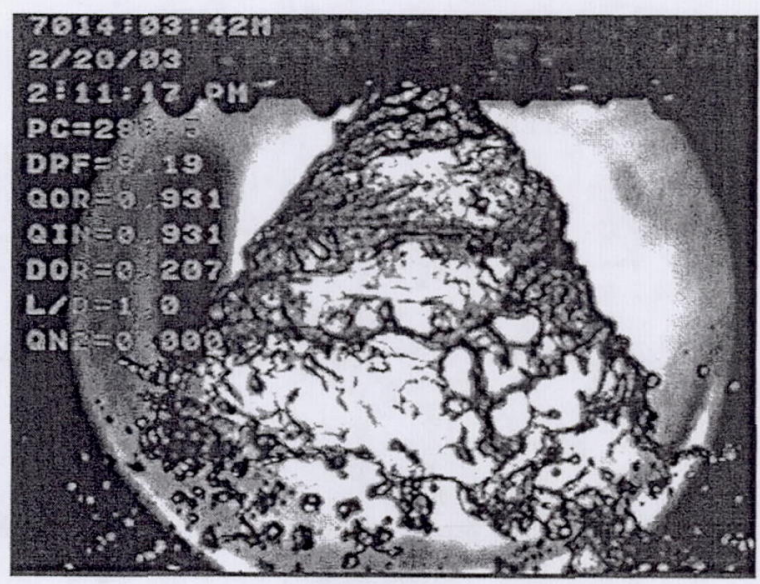

Figure 3: Back Lit Photography of Differential Velocity Test Series Spray, $\mathrm{Pc}=300 \mathrm{psia}, \mathrm{N}_{2}=0 \mathrm{lb} / \mathrm{s}$ Flow, $\mathrm{H}_{2} \mathrm{O}=0.16 \mathrm{lb} / \mathrm{s}$

However, this conical sheet quickly collapses when the gas flow is turned on. As little as $\mathrm{N}_{2}=0.07 \mathrm{lb} / \mathrm{s}$ was required to collapse the gas/liquid spray like seen in Figure 5.

For the low pressure 300 psia survey, gas flow rates were varied between $0.2-0.6 \mathrm{lb} / \mathrm{s}$, with liquid flow rates being varied between $0.06-0.19 \mathrm{lb} / \mathrm{s}$ to maintain a gas to liquid ratio of $3.15: 1$.

Most of the conditions produced a symmetric and uniform spray as evidenced by patternation data presented in Figure 6. A slight asymmetry can be observed for the $\mathrm{N}_{2}=0.2 \mathrm{lb} / \mathrm{s}$ case on the left of Fig. 6 , however this asymmetry can be attributed to the poor film formation attributed to the low pressure drop at that condition. 


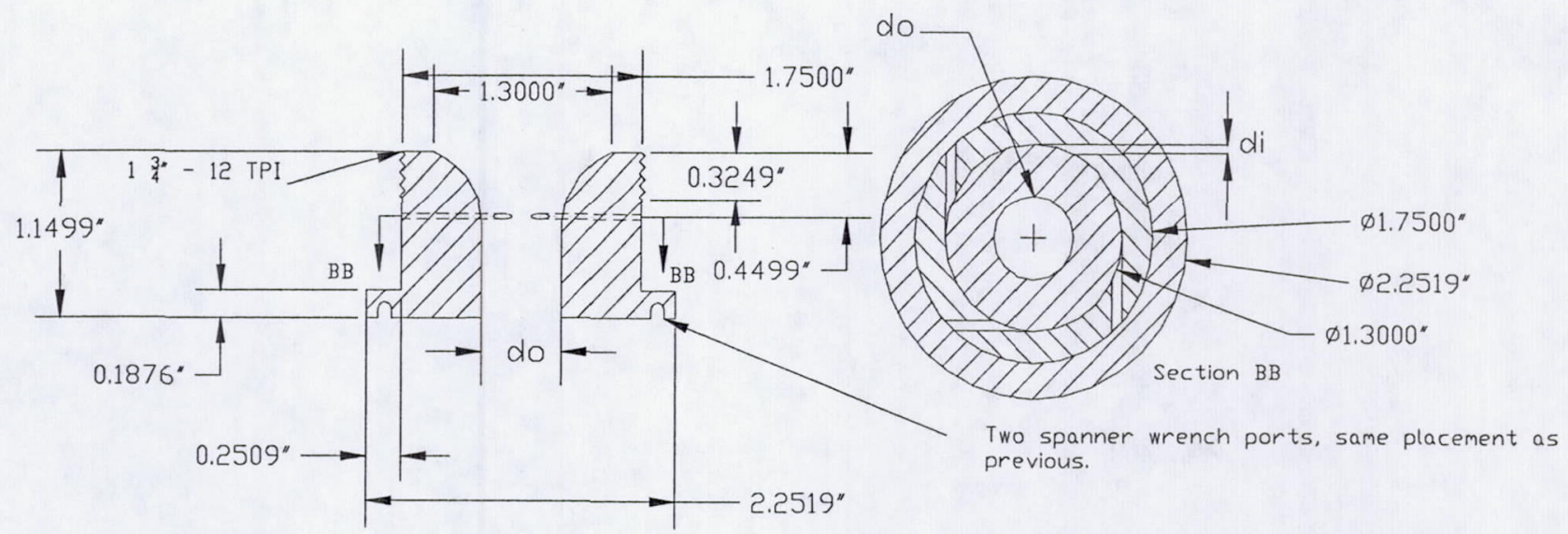

Figure 4: Differential Velocity Survey Injector for Cold Flow Testing*

*Variations described in Table 4

Table 4: Summary of Differential Velocity Injector Dimensions

\begin{tabular}{|c|c|c|}
\hline Injector Chamber Pressure (psia) & di (in) & do (in) \\
\hline 300 & 0.0718 & 0.2193 \\
\hline 1150 & 0.0844 & 0.4312 \\
\hline
\end{tabular}

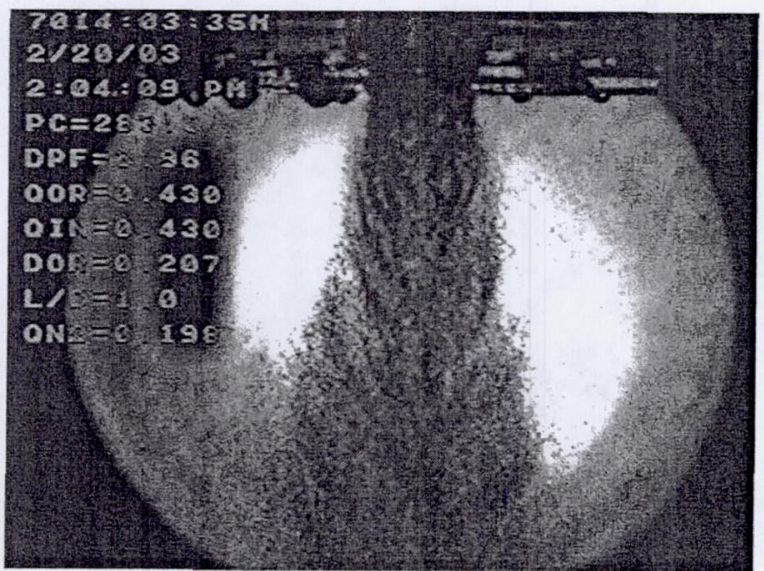

Figure 5: Back Lit Photography of Differential Velocity Test Series Spray, Pc $=300$ psia, N2 $=0.2 \mathrm{lb} / \mathrm{s}$, $\mathrm{H}_{2} \mathrm{O}=0.06 \mathrm{lb} / \mathrm{s}$.

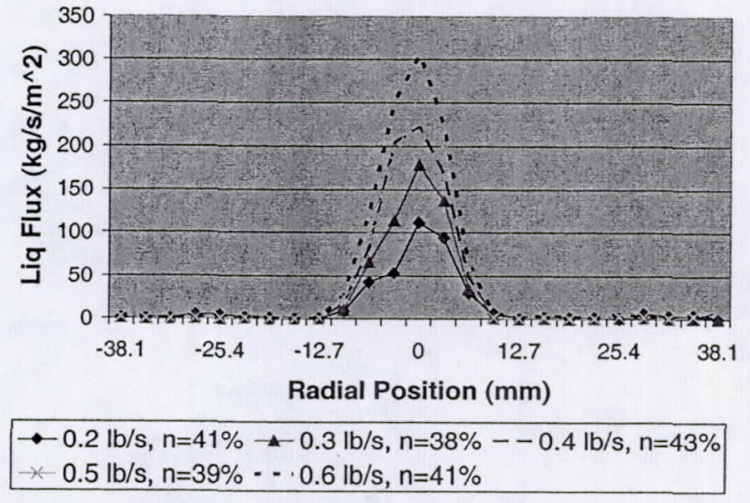

Figure 6: Patternation Data for $\mathrm{Pc}=300$ psia Differential Velocity Test Series, 1/8" Patternator (1x27); Collection Efficiency Reported Next to Series N2 Mass Flow Rate 


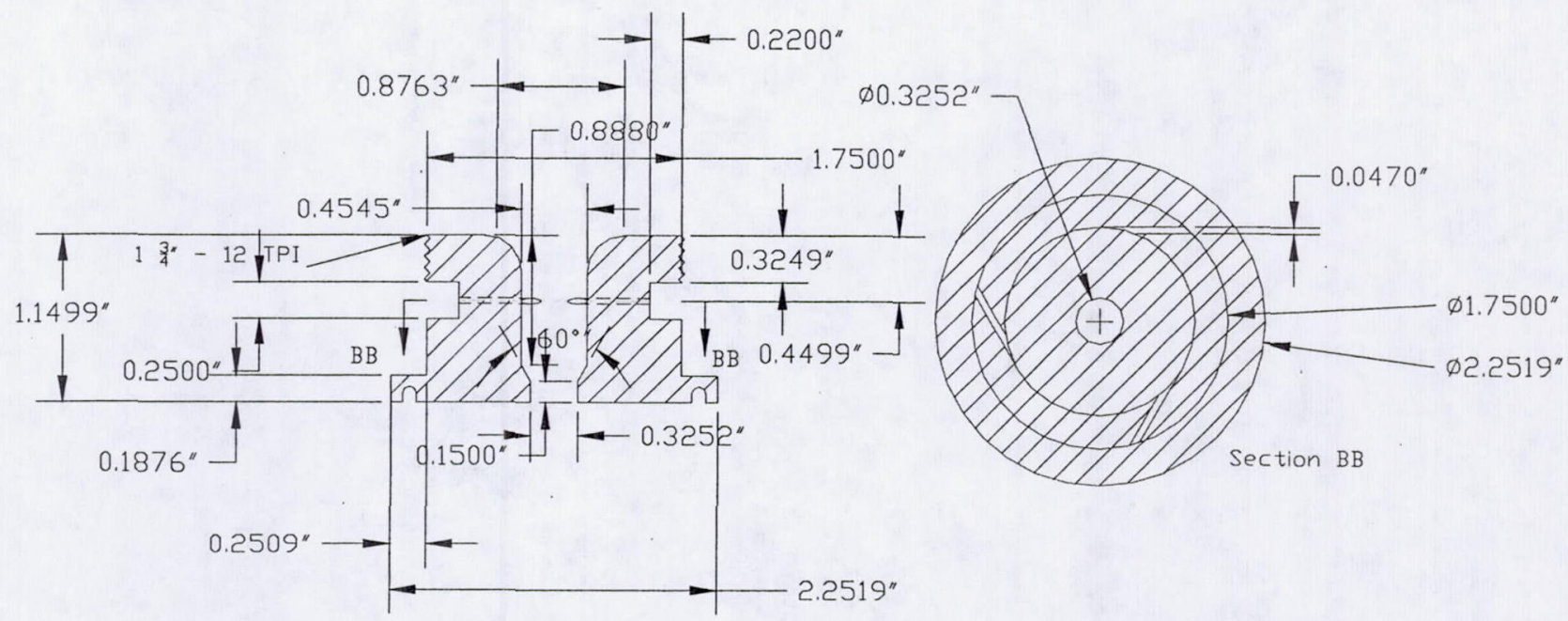

Figure 7: Momentum Flux Ratio Survey Injector for Cold Flow Testing

The PDPA was run for the lower flow rate conditions. Results showed that even with the slightly asymmetry shown by the patternator, fine drops on the order of $65 \mu \mathrm{m}$ were produced. A typical plot of the Sauter Mean Diameter $\left(\mathrm{d}_{32}\right)$ and velocity versus radial position in the spray is shown in Figure 8. The reduction in $d_{32}$ as the PDPA was swept radially across the flow was due to mostly to the positioning of the intersecting laser beams between the increasingly dense spray and the detector, obscuring the light detector.

Measurements at higher flow rates and velocities proved to be problematic as the sprays became increasingly dense, as seen in Figure 9. The sprays would overwhelm the patternator, and prohibited accurate measurements of the drop size diameters at the center of the spray. While some measurements could be taken for the lower $\mathrm{P}_{\mathrm{c}}$ differential velocity survey, the fine atomization and dense sprays of the high $P_{c}$ test series permitted only the back-lit photography.

A momentum flux ratio survey was conducted by fixing the injector geometry and mass flow rate. The MFR was altered in the chamber by adjusting the chamber pressure. The pressure change would alter the nitrogen density and hence the velocity of the gas. Back-lit photography was first used to initially assess the sprays in the MFR survey. As testing progressed, while the sprays were finely atomized and uniform, it became apparent that there was little to no significant change in the spray as the MFR was occurring. The differential velocity survey yielded much more insightful results than the MFR series, indicating the importance of this parameter as shown in Eqs. (2-5).

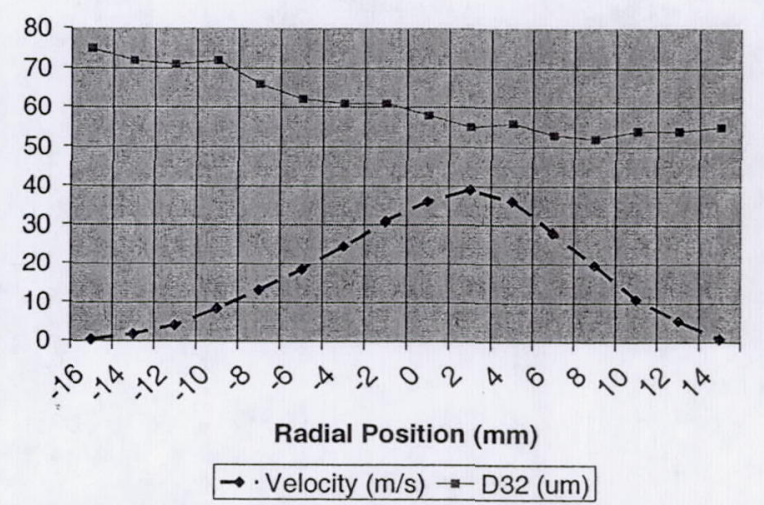

Figure 8: PDPA Data for $\mathrm{Pc}=300$ psia Differential Velocity Test Series, $\mathrm{N}_{2}=0.2 \mathrm{lb} / \mathrm{s}, \mathrm{H}_{2} \mathrm{O}=0.06 \mathrm{lb} / \mathrm{s}$, Intensity Validation $65 \%$.

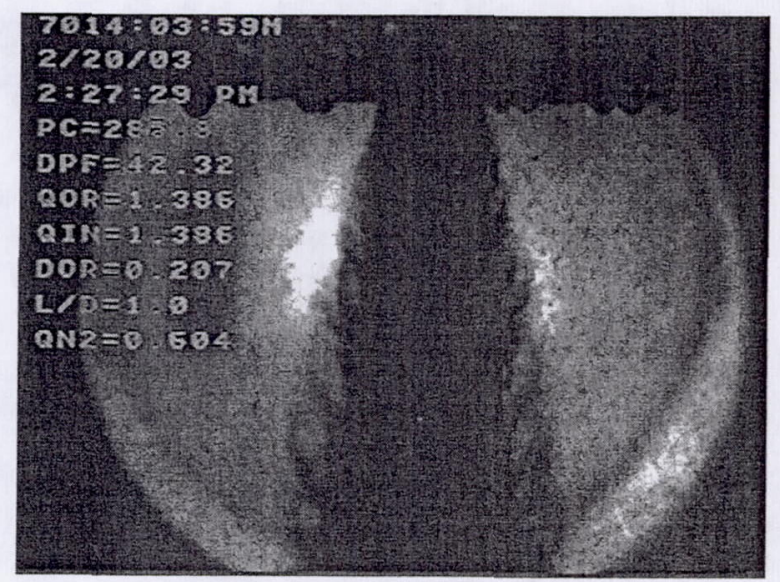

Figure 9: Back Lit Photography of Differential Velocity Test Series Spray, Pc=300 psia, N2=0.6 lb/s, $\mathrm{H}_{2} \mathrm{O}=0.19 \mathrm{lb} / \mathrm{s}$ 


\section{SUMMARY AND CONCLUSIONS}

A set of operating conditions and general design features for a baseline gas-centered, liquid swirl injector have been chosen based on open literature sources. At this initial stage of the study, it is speculated that the baseline gas-liquid injector element will be practically insensitive to pressure and velocity pulsations in the combustion chamber. Further analysis and experimentation is required. It does not produce self-oscillations, nor is it sensitive to pressure drop pulsations applied to liquid propellant injector stage. The gas part of the injector comprises an acoustic resonator tuned to withdraw a significant part of acoustic energy from the combustion chamber to the gas manifold, where it is can be dissipated by perforated plates. The atomization process which uses high gas densities and a rotating liquid film is efficient and provides even mixing. Remnants of the liquid fuel film protects the injector face from overheating. The ability to increase the thickness of the combustion zone as the amplitude of the pressure drop pulsations grows allows servo-stabilization of combustion instability. Cold flow testing has been conducted, and shows fine atomization and symmetric sprays. Difficulties arose using patternation and PDPA techniques on sprays at high mass flow rates/chamber pressures as the sprays were too dense to permit accurate measurements.

\section{ACKNOWLEDGEMENTS}

This work was funded by NASA Marshall Space Flight Center, Grant NAG8-1894. The authors wish to acknowledge Dr. Terri Tramel and Mr. Joseph Sims of Marshall for their help and guidance. The authors would also like to acknowledge Dr. Pete Strakey of AFRL for his advice and time in helping conduct cold flow experiments. Mr. Curtis Johnson and Dr. Jeff Muss of Sierra Engineering are acknowledged for their extensive collaboration and assistance in starting this effort. The authors also acknowledge Mr. Larry Jones of Medtherm for his consultation on thermal measurements for single element testing, and Messrs. Scott Meyer and James Sisco of Purdue for their helpful suggestions with the injector design.

\section{REFERENCES}

1. Anon., Aviation, Rocket, Marine and Industrial Engines of 1944-2000. Moscow, AKSKonversalt, 2000.

2. Semenov, J., Buran Spacecraft, Mashinostroenie, 1996.
3. Harrje, D.T., and Reardon, F.H., Liquid Propellant Rocket Combustion Instability, NASA SP-194, 1972.

4. Orlov, V.A., "Stationary and dynamic characteristics of shear-coaxial injectors for staged combustion cycle engines," $\mathrm{Ph}$. D. Thesis, NIICHIMMASH, Russia, 2002.

5. Bazarov, V., "Self-Pulsations in Coaxial Injectors with Central Swirl Liquid Stage," AIAA Paper No. 95-2358, $31^{\text {st }}$ AIAA/ASME/SAE/ASEE Joint Propulsion Conference \& Exhibit, San Diego, CA, July 10-12, 1995.

6. Andreev, A., Chepkin, V., and Fanciullo, T., "The Development of the D-57 Advanced Staged Combustion Engine for Upper Stages," AIAA Paper No. 94-3378, June 1994.

7. Muss, J.A., Johnson, C.W., Cohn, R.K., Strakey, P.A., Bates, R.W., Talley, D.G., "Swirl Coaxial Injector Development Part I - Test and Results," $38^{\text {th }}$ JANNAF Combustion Subcommittee Meetings, Destin, FL, 8-12 April, 2002.

8. Cheng, G., Johnson, C.W., and Muss, J.A., "Swirl Coaxial Injector Development Part II - CFD Modeling," $\quad 38^{\text {th }} \quad$ JANNAF Combustion Subcommittee Meetings, Destin, FL, 8-12 April, 2002.

9. Manski, Detlef, et al., "Cycles for Earth-to-Orbit Propulsion," Journal of Propulsion and Power, vol. 14, no. 5, Sept.-Oct. 1998, p. 588-604.

10. Sutton, George, Biblarz, Oscar, Rocket Propulsion Elements $7^{\text {th }} E d$, Wiley-Interscience, 2000.

11. Vasin, A., et al., United States Patent, US 6,244,041, B1, Jun. 12, 2001.

12. Long, M.R., Anderson, W.E., Humble, R.W., "BiCentrifugal Swirl Injector Development for Hydrogen Peroxide and Non-Toxic Hypergolic Miscible Fuels", AIAA Paper 2002-4026, 38th AIAA/ASME/SAE/ASEE Joint Propulsion Conference \& Exhibit, Indianapolis, $\mathbb{N}$, July 7-10, 2002.

13. Bayvel, L., Orzechowski, Z., Liquid Atomization, Taylor \& Francis, 1993.

14. Bazarov, V. and Yang, V., "Liquid-Propellant Rocket Injector Dynamics," Journal of Propulsion and Power, Vol. 14, \# 5, September - October 1998, pp. 797-806.

15. Andreev, A., Bazarov, V., Dushkin, A., Lul'ka, L., and Grigoriev, S. Dynamics of gas-liquid injectors. M., Mashinostroenie, 1991.

16. Bazarov, V., Dynamics of Liquid Injectors, Moscow, Mashonostroenie, 1979.

17. Bazarov, V., "Influence of Propellant Injector Stationary and Dynamic Parameters on High Frequency Combustion Stability,“ AIAA Paper No. $96-3119,32^{\text {nd }}$ AIAA/ASME/SAE/ASEE Joint Propulsion Conference \& Exhibit, July 1-3, 1996. Lake Buena Vista, FL. 